\title{
Variation du Vent et ses Influences sur les Parametres Climatiques de la Région de Yangambi (RD Congo)
}

\author{
Likoko B. A., Pédologue \\ Likoko G. A., Phyto-technicien \\ Mbifo N. J., Agro-climatologiste \\ Besango L., Agro-climatologiste \\ Inera Yangambi
}

Doi:10.19044/esj.2020.v16n9p196 ～URL:http://dx.doi.org/10.19044/esj.2020.v16n9p196

\section{Resume}

La région forestière de Yangambi subi des perturbations climatiques suite au réchauffement global. Ainsi à cause de degâts entrainés par des tempêtes, la présente étude a été menée pour déterminer par le test de Pettit et de Welch le changement tendanciel sérial du vent et son influence par les coefficients de corrélations simples et partielles sur les paramètres climatiques observés de 1950 à 2017 à Yangambi. Il s'est avéré que l'augmentation de la température à $24,98 \pm 0,15^{\circ} \mathrm{C}$ a influencé la hausse significative depuis 1990 de la tendance du vent d'une vitesse moyenne annuelle de $2,85 \pm 0,17 \mathrm{~km} / \mathrm{h}$. Les grandes vitesses mensuelles du vent ont été observées en mars avec 3,16 $\mathrm{km} / \mathrm{h}$ et octobre avec $2,99 \mathrm{~km} / \mathrm{h}$. L'évapotranspiration potentielle (ETP) et la chaleur latente étant très élevées en mars et octobre, influencent la baisse de l'humidité relative de l'air (HR) et produisent des grandes variations de vent pour déclencher les premières pluies aléatoires. La relation mensuelle simple du vent a été significative avec tous les paramètres climatiques. Le vent a été hautement influencé par la corrélation mensuelle partielle de $\mathrm{r}=-0,918$ entre la température et l'HR moyenne de $87,3 \%$ observées dans la divergence symétrique de leurs régimes, et $\mathrm{r}=0,698$ entre la température et l'ETP moyenne de 95,3 $\mathrm{mm}$ observées dans la convergence symétrique de leurs coefficients de variation.

Mots clés: Vent, Changement De Tendance, Paramètres Climatiques, Correlation Et Yangambi 


\title{
Wind Variation and Influences on the Climatic Parameters of Yangambi Region (RD Congo)
}

\author{
Likoko B. A., Pédologue \\ Likoko G. A., Phyto-technicien \\ Mbifo N. J., Agro-climatologiste \\ Besango L., Agro-climatologiste \\ Inera Yangambi
}

\begin{abstract}
The forest region of Yangambi has met climatic disturbances because of global warming. Thus, for disasters caused by tempests, the present study has been conducted to determine by the test of Pettit and Welch the serial trend change of wind and its influence by the coefficients of simple and partial correlations on the climatic parameters registered from 1950 to 2017 in Yangambi. It has been revealed that the temperature increase at $24.98 \pm 0.15^{\circ} \mathrm{C}$ has influenced since 1990 the significant increase of annual wind trend with the speed means of $2.85 \pm 0.17 \mathrm{~km} / \mathrm{h}$. Higher monthly wind speeds have been observed in march with $3.16 \mathrm{~km} / \mathrm{h}$ and october with $2.99 \mathrm{~km} / \mathrm{h}$. Potential evapotranspiration (PET) and latent hit, being very high in march and october, influence the declining of air relative humidity $(\mathrm{RH})$ and yield high wind variations that could enhance aleatory first rainfall. Monthly simple correlationship of wind has been significant with all climatic parameters. The wind has been highly influenced by the monthly partial correlation with $\mathrm{r}=$ 0.918 between temperature and $\mathrm{RH}$ averaged at $87.3 \%$ observed in the symmetrical divergence of their regimes and $\mathrm{r}=0.698$ between temperature and PET averaged at $95.3 \mathrm{~mm}$ following the symmetrical convergence of their coefficients of variation.
\end{abstract}

Keywords : Wind, Trend Change, Climatic Parameters, Correlation And Yangambi

\section{Introduction}

Le changement climatique modifie la circulation atmospherique (Quentin, 2018). A Yangambi, les perturbations pluviometriques mensuelles et les changement serials de temperatures, de l'humidite relative de l'aire et de l'ETP ont ete observées (Likoko et al., 2019a, 2019b). Malgré l'accroissement significatif de température moyenne de $0,45^{\circ} \mathrm{C}$ à partir de 1980 , le climat 
chaud et humide a persisté en maintenant stationnaires les pluies annuelles, mais en subissant des perturbations de précipitations mensuelles. Les pluies ont accru en grande saison humide d'août à novembre avec $87 \%$ d'humidité relative de l'air, pendant que la sècheresse est accentuée en grande saison sèche de décembre à février avec la diminution de l'HR à $82 \%$ (Likoko et al., 2019a). Par contre les valeurs annuelles de l'humidité relative de l'air et de l'ETP ont subi une rupture de changement significatif négatif observé en 1975 et 1984 repectivement, contrairement à la tendance ascendante de températures (Likoko et al., 2019b).

De tous les facteurs atmosphériques étudiés, le vent n'est pas encore mis en évidence pour sa vitesse d'action. A Yangambi, les vents de fortes ampleurs causent des verses des cultures cérealières et bananières, véhiculent les matériels génétiques végétaux (pollens, graines) et les maladies phytopathologiques, font tomber des fleurs, abattent et élaguent des arbres dahs la réserve biosphérique, detruisent les édifices, détolent les maisons et entrainent des naufrages et noyades sur le fleuve Congo (Boyemba, 2011 ; INERA, 2017).

D'après l'équation de Penman-Monteith, le vent est le mouvement des masses d'air qui se déplacent à cause de la différence de pressions créée par la différence de températures d'une zone de hautes pressions vers une zone de basses pressions de l'atmosphère, en fonction de l'altitude et du relief diminuant ainsi l'humidité relative de l'air et élevant l'ETP pour faire tomber la pluie (Allen et al., 1998). L'objectif de la présente étude est de déterminer le changement tendanciel sérial du vent et ses relations avec les élements climatiques de l'air à Yangambi où le phénomène de convection atmosphérique est principal (Bultot, 1957).

\section{Zone d'étude et données}

\subsection{Localisation de la zone d'étude}

La région écologique de Yangambi couvre 440.000 hectares situés à $0^{\circ} 49^{\prime}-0^{\circ} 54^{\prime} \mathrm{N}, 24^{\circ} 29^{\prime}-24^{\circ} 49^{\prime} \mathrm{E}$ et $435-485 \mathrm{~m}$ d'altitude dans la cuvette centrale africaine de la RD Congo. Les sols sont ferralitiques végétés par la forêt dense humide sempervirente ombrophile à Brachystesia laurentii, caducifoliée à Scorodophleus zenkeri et Pericopsis elata et semi-caducifoliée à Gilbetiodendron dewevrei (Kearsly, 2015; Alongo et al., 2013). 


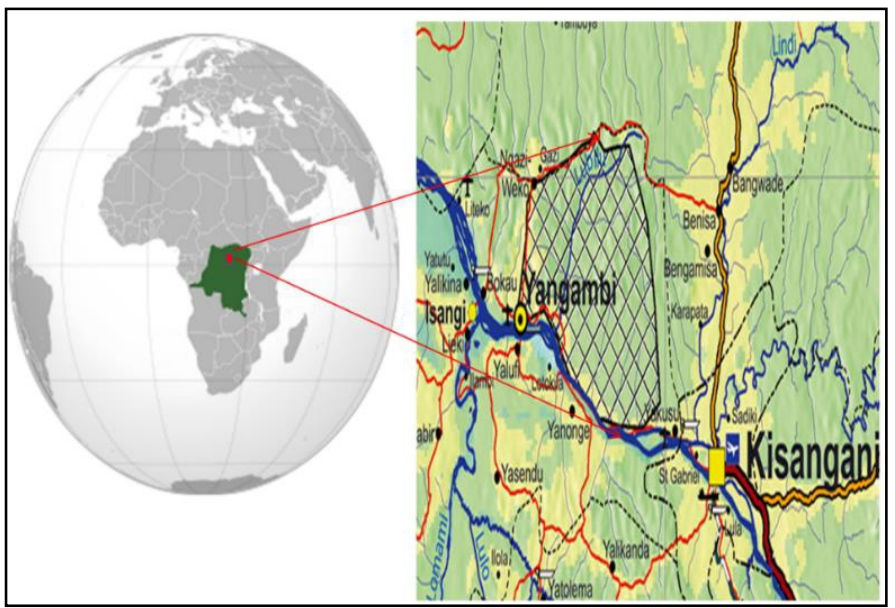

Figure 1 : Le Centre de recherche INERA Yangambi (en boule jaune) et sa réserve écologique de forêt dense humide sempervirente quadrillée au nord de la RDC (en rouge) en Afrique centrale (Source, Google map, 2016).

Le régime mensuel à Yangambi a défini le climat chaud et humide persévérant face au réchauffement global. Il est actuellement caractérisé par la double périodicité des pluies de deux maxima aux équinoxes de mai et octobre, et deux minima aux solstices de juin et de janvier. L'ETP forme aussi deux minima de juin à août et de novembre à décembre, et deux maxima en octobre le secondaire et en mars le principal. Décembre est le mois le moins évapo-transpirant avec $90,5 \mathrm{~mm}$ et mars, le mois le plus évapo-transpirant avec 102,61 mm. D'autre part, les mois de février et de mars sont les plus chauds avec respectivement les températures maxima de $31,22^{\circ} \mathrm{C}$ et $31,03^{\circ} \mathrm{C}$. Les plus faibles températures maxima se retrouvent en juillet avec $28,77^{\circ} \mathrm{C}$ et en août avec $28,84{ }^{\circ} \mathrm{C}$ (Likoko et al., 2019a). La vitesse moyenne mensuelle du vent oscille entre $2,56 \mathrm{~km} / \mathrm{h}$ en janvier et décembre, et $3,14 \mathrm{~km} / \mathrm{h}$ en mars.

\subsection{Données de l'étude}

Les données d'observations journalières sur la vitesse du vent (exprimé en $\mathrm{km} / \mathrm{h}$ ), l'humidité relative de l'air (HR exprimée en \%), l'évapotranspiration potentielle (ETP exprimée en $\mathrm{mm}$ ) et les températures moyennes (exprimées en dégrée Celsius, ${ }^{\circ} \mathrm{C}$ ) recueillies de 1950 à 2017 à la station climatologique de $\mathrm{Km} 5$ de l'INERA Yangambi, ont été traitées en valeurs moyennes mensuelles et annuelles.

\section{Méthodes}

\subsection{Méthodes d'identification de fluctuations et test de tendance}

Les fluctuations des paramètres climatiques ont été déterminées par les coefficients de variation (CV \%) et de corrélations simples et partielles (r) nonparamétriques de Spearman's rho calculés par rapport au vent. Les moyennes 
des paramètres ont été statistiquement et graphiquement analysées avec le logiciel R 3.1 pour trouver les variations de tendance par les tests de non stationnarité Wald Wolfowitz et de Mann Kendhall (Thorsten, 2018).

\subsection{Tests de détection des ruptures}

Le point de changement climatique annuel était détecté suivant le test de Pettitt (1974) et la méthode statistique U de Buishand (1984). Le symbole $\mathrm{z}$ dans le test représente le coefficient standardisé pour la régression et $\mathrm{U}$ représente le degré de rupture de série. Le test de Welch a comparé les séries de rupture.

\section{Resultats}

\subsection{Variations Mensuelles Des Éléments Climatiques De L'air}

Les régimes de variations mensuelles de la vitesse du vent, de l'humidité relative de l'air, de l'ETP et des températures sont présentés dans la figure 2 .

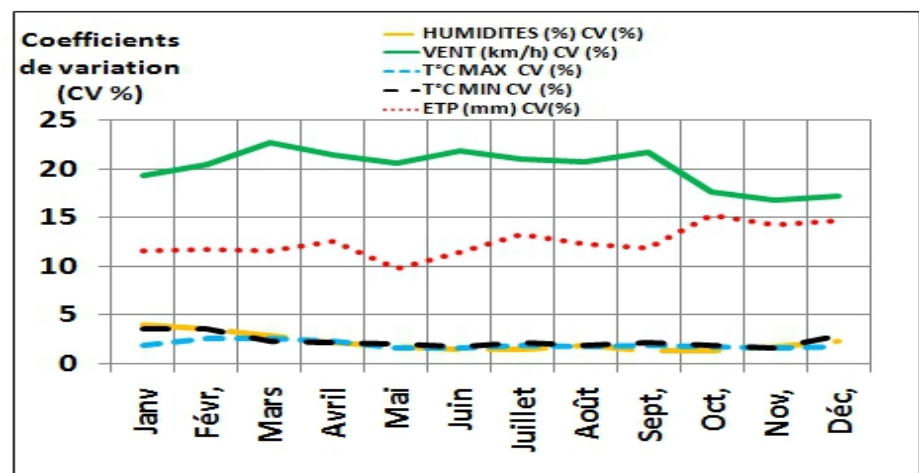

Figure 2 : Coefficients de variation (\%) de la vitesse du vent, l'ETP, l'humidité relative de l'air et les températures maxima et minima observés de 1950 à 2015 à Yangambi,

RDC (Source : Station climatologique Km5 INERA Yangambi, 2019)

En figure 2, les coefficients de variation des vitesses du vent, de février à septembre, ont été supérieurs à $20 \%$ dont les plus élevés en mars avec 23 $\%$, juin $22 \%$ et septembre $21,5 \%$, pendant que d'octobre à décembre, ils étaient les plus bas de $\pm 17 \%$. Par contre, les coefficients de variation les plus élevés de l'ETP ont été observés en octobre, novembre et décembre respectivement avec $15,24 \%, 14,18 \%$ et $14,69 \%$ contre le mois de mars avec $9,68 \%$. Les valeurs d'humidité les plus élevées supérieures à $87 \%$ ont été observées de juin à décembre, ayant humidifié la grande saison humide en moyenne de $87,88 \%$, où le CV a été le plus faible de 2 à $4 \%$. Les CV de températures maxima et minima ont été aussi faibles de 2 à $4 \%$, pourtant elles sont les plus influentes. 


\subsection{Relations mensuelles du vent avec les éléments climatiques de l'air}

Les relations mensuelles entre le vent et les températures de l'air, l'ETP et l'humidité relative de l'air observées à Yangambi de 1950 à 2017 sont présentées au tableau 1.

Tableau 1 : Les coefficients mensuels de corrélations simples et partielles des éléments climatiques de l'air observés de 1950 à 2017 à Yangambi.

\begin{tabular}{|c|c|c|c|c|c|c|c|c|c|}
\hline \multicolumn{6}{|c|}{ 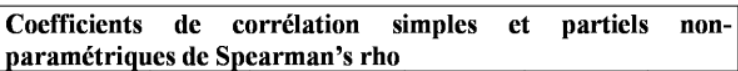 } & \multicolumn{4}{|c|}{$\begin{array}{l}\text { Coefficients de corrélation } \\
\text { partiels du vent constant }\end{array}$} \\
\hline & VENT & $\begin{array}{l}\text { HUMIDITE } \\
\text { RELATIVE }\end{array}$ & ETP & $\begin{array}{l}\text { TEMP. } \\
\text { MAX }\end{array}$ & $\begin{array}{l}\text { TEMP. } \\
\text { MIN }\end{array}$ & $\begin{array}{c}\text { HUMID } \\
\text { ITE }\end{array}$ & ETP & $\begin{array}{l}\text { TEMP. } \\
\text { MAX }\end{array}$ & $\begin{array}{l}\text { TEMP. } \\
\text { MIN }\end{array}$ \\
\hline VENT & 1 & & & & & & & & \\
\hline $\begin{array}{l}\text { HUMIDITE } \\
\text { RELATIVE }\end{array}$ & $-0,522^{*}$ & 1 & & & & 1 & $-0,663^{*}$ & $-0,918^{* *}$ & $-0,194$ \\
\hline ETP & $0,634^{*}$ & $-0,762 * *$ & 1 & & & & 1 & $0,693^{* *}$ & 0,400 \\
\hline TEMP. MAX & $0,501^{*}$ & $-0,937 * *$ & $0,706 * *$ & 1 & & & & 1 & $\begin{array}{c}0,523^{*} \\
*\end{array}$ \\
\hline TEMP. MIN & $0,526^{*}$ & $-0,473$ & 0,378 & $0,687^{* *}$ & 1 & & & & 1 \\
\hline
\end{tabular}

Légende: *significatif au point de probabilité 0,05, **hautement significatif au point de probabilité 0,05 (Source : Station climatologique Km5 INERA Yangambi, RDC 2019)

La relation mensuelle simple du vent avec les éléments climatiques de l'air a été significative au point 0,05 de probabilité, notamment avec l'humidité relative de l'air $(\mathrm{r}=-0,522)$, l'ETP $(\mathrm{r}=0,634)$, la température maximum $(r=0,501)$ et la température minimum $(r=0,526)$.

En outre, les coefficients mensuels de corrélations simples hautement significatifs ont été observés entre l'humidité relative de l'air avec la température maximum et l'ETP respectivement avec $\mathrm{r}=-0,762$ et $\mathrm{r}=-0,937$. L'ETP et la température maximum ont hautement corrélé avec $r=+0,706$. La corrélation simple entre les deux températures a été hautement significative avec $\mathrm{r}=+0,687$.

La corrélation partielle mensuelle du vent maintenu constant a été hautement influencée par la température maximum avec l'humidité relative et l'ETP respectivement au $\mathrm{r}=-0,918$ et $\mathrm{r}=0,693$; pendant que $\mathrm{r}$ était significativement égale à $-0,665$ entre l'ETP et l'humidité relative de l'air.

\subsection{Relations Annuelles du vent avec les éléments climatiques de l'air}

Les relations annuelles entre le vent et les températures de l'air, l'humidité relative de l'air et l'ETP observées à Yangambi de 1950 à 2017, sont présentées au tableau 2. 
Tableau 2 : Les coefficients annuels de corrélations simples et partielles des éléments climatiques de l'air observés de 1950 à 2017 à Yangambi.

\begin{tabular}{|c|c|c|c|c|c|c|c|c|c|}
\hline \multicolumn{6}{|c|}{$\begin{array}{l}\text { Coefficients de corrélation simples et partiels non- } \\
\text { paramétrique de Spearman's rho. }\end{array}$} & \multicolumn{4}{|c|}{$\begin{array}{l}\text { Coefficients de corrélation } \\
\text { partiels du vent constant. }\end{array}$} \\
\hline & VENT & HUM & ETP & $\begin{array}{l}\text { TEMP. } \\
\text { MAX }\end{array}$ & $\begin{array}{l}\text { TEMP. } \\
\text { MIN }\end{array}$ & $\begin{array}{l}\text { HUM } \\
\text { IDIT } \\
\text { E }\end{array}$ & ETP & $\begin{array}{l}\text { TEMP. } \\
\text { MAX }\end{array}$ & $\begin{array}{l}\text { TEMP. } \\
\text { MIN }\end{array}$ \\
\hline VENT & 1 & & & & & & & & \\
\hline $\begin{array}{l}\text { HUMIDITE } \\
\text { RELATIVE }\end{array}$ & $-0,1$ & 1 & & & & 1 & 0,039 & $-0,449 * *$ & $-0,181$ \\
\hline ETP & $0,297^{\star}$ & 0,023 & 1 & & & & 1 & $-0,063$ & $-0,053$ \\
\hline TEMP. MAX & $-0,580^{\star *}$ & $-0,385^{\star *}$ & $-0,197$ & 1 & & & & 1 & 0,682 * * \\
\hline TEMP. MIN & 0,164 & $-0,455 *$ * & 0,102 & $0, \mathbf{5 3 9} 9^{* *}$ & 1 & & & & 1 \\
\hline
\end{tabular}

Légende : *significatif au point de probabilité 0,05, **hautement significatif au point de probabilité 0,05 (Source : Station climatologique Km5 INERA Yangambi, RDC 2019).

La température maximum a hautement influencé négativement le vent et l'humidité de l'air avec $\mathrm{r}=-0,580$ et $-0,385$. Par contre, elle a influencé positivement la température minimum avec $\mathrm{r}=+0,539$, pendant que la température minimum et l'humidité relative exprimaient la corrélation hautement significative avec $\mathrm{r}=-0,455$.

En maintenant le vent constant, la corrélation partielle annuelle hautement significative $r=-0,449$ a été observée entre l'humidité relative de l'air et la température maximum et $r=0,682$ entre la température maximum et la température minimum.

\subsection{Variation du vent}

Le test Wald - Wolfowitz montre que la série du vent annuel pendant 47 ans n'a pas été stationnaire avec $p$-value $=2.88 \mathrm{e}-08$ et $\mathrm{z}=5,5486.3$. De la figure 3, la rupture de la série détecté à 19 ans en 1990, a été significative avec $p$-value $=2.25 \mathrm{e}-06$ et $\mathrm{U}=492$.

La figure 3 montre que le test de Welch sur le vent présente la différence significative entre la première sous série A descendant de 1971 à 1990 à la vitesse moyenne annuelle de $3,234 \mathrm{~km} / \mathrm{h}$ et la seconde sous série B ascendant de 1991 à 2017 à la vitesse moyenne annuelle de 2,466 km/h de $p$ value $=2.175 \mathrm{e}-09$ et $\mathrm{t}=7,4671$ à $\mathrm{df}=44,659$. 


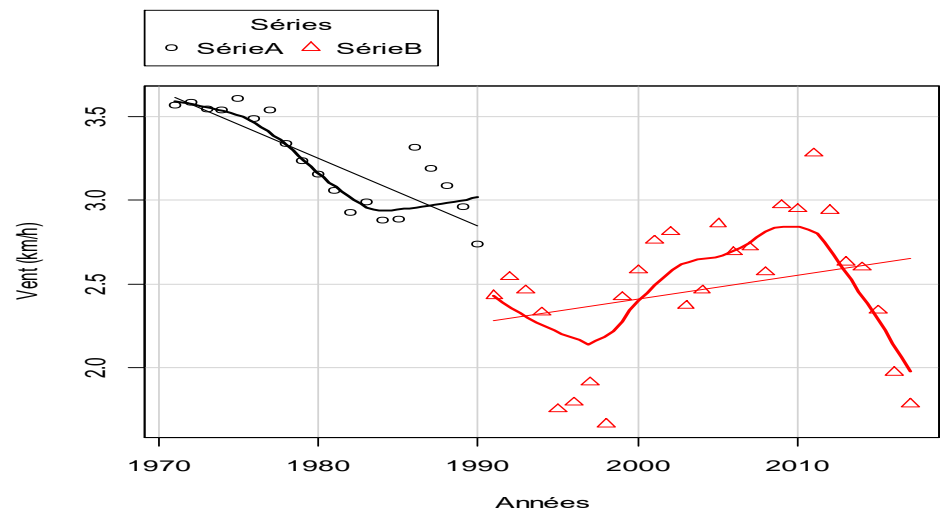

Figure 3 : Point de rupture en sous série A de 1971-1990 et sous série B de 1991-2017 de la chronologie du vent de 1971 à 2018 à Yangambi (Source : Station climatologique km5

INERA Yangambi, RDC 2019).

\section{Discussion}

L'effet de chaleur globale à Yangambi se confirme par le changement de tendance de température moyenne détecté en 1977 qui a été hautement significatif $(\mathrm{p}<0,001)$ par l'augmentation de $0,44^{\circ} \mathrm{C}$ (Likoko et al., 2019b). Cette élévation significative de la température moyenne du régime annuel a abaissé significativement la vitesse du vent de 1950 à 1990, hautement corrélées par le coefficient de corrélation $\mathrm{r}=-0,580$. L'intensification de l'ETP et des précipitation suite au réchauffement climatique, a provoqué une accumulation d'air humide ayant diminué la vitesse du vent de la circulation atmosphérique (Paeth et al., 2013 ; Quentin, 2018). De 1990 à 2017, la sous série du vent annuel est ascendante influencée par la hausse de température telle qu'observée sur les océans par Young et al. (2018).

Quant au régime mensuel, l'augmentation de température moyenne est corrélée à l'augmentation de la vitesse du vent avec $\mathrm{r}=0,501$ répondant de ce fait à l'équation de Penman-Monteith, où le vent exprimé en termes de déficit de pressions de l'air fonctionne avec la température, la capacité thermique de l'air, l'humidité de l'air, la latitude et l'altitude comme paramètres de l'évapotranspiration réelle de référence (Allen et al., 1998).

Contrairement au vent et à la température, les tendances annuelles négatives pour l'humidité relative de l'air et de l'ETP ont été rompues à la baisse (Likoko et al., 2019b). Ceci conclu l'existence des perturbations tendancielles des éléments climatiques.

A Yangambi, malgré ses faibles valeurs moyennes mensuelles, les vents journaliers de mars et d'octobre causent des dégâts par son phénomène de convection atmosphérique, précédant les pluies (Bultot, 1957). En grande saison sèche, l'ETP est supérieure aux précipitations et les grandes températures de cette période ayant développé des déficits de saturation d'eau 
dans le sol et dans l'atmosphère, résultent une ETP et une chaleur latente élevées en mars où se manifestent le pic principal d'ETP, les grandes variations de vents et la dépression d'humidité relative pour déclencher les premières pluies aléatoires de la petite saison pluvieuse. Les vents mensuels de Yangambi sont des brises légères d'après l'échelle de Beaufort (Hirsch et al., 2016).

\section{Conclusion}

Le changement tendanciel sérial du vent et ses relations avec les paramètres climatiques de la région de Yangambi ont été déterminés. Les résultats montrent que la vitesse du vent est plus élévée en mars avec 3,16 $\mathrm{Km} / \mathrm{h}$ et octobre avec $2,99 \mathrm{~km} / \mathrm{h}$. Le changement tendanciel du vent à la hausse a été détecté en 1990, influencé par l'augmentation de la température au coefficient de corrélation $\mathrm{r}=0,501$ et de l'ETP au $\mathrm{r}=0,634$, et par la diminution de l'humidité relative de l'aire avec $r=-0,522$. L'étude de jours de vent en mars et octobre éluciderait davantage le temps précis de tempêtes causant de dégâts.

\section{References:}

1. Allen R.G., Pereira L.S., Raes D. et Smith M. (1998). Crop Evapotranspiration-Guidelines for Computing Crop Water Requirements. FAO Irrigation and drainage paper 56. Rome, Italy: Food and Agriculture Organization of the United Nations. ISBN 925-104219-5. http://www.fao.org/docrep/X0490E/x0490e00.htm. Retiré le 20 novembre 2019.

2. Alongo S., Visser M., Kombele F., Colinet C. et Bogaert J. (2013). Propriétés et diagnostic de l'état agropédologique du sol de la série Yakonde après fragmentation de la forêt à Yangambi, R D Congo. Annales des instituts supérieurs d'études agronomiques, 2013, 5(1), pp36-51. <hal-00875748>

3. Boyemba F. (2011). Ecologie de Pericopsis elata (Harms) Van Meeuwen (Fabaceae), arbre de forêt tropicale africaine à répartition agrégée. Thèse de doctorat, université libre de Bruxelles. 181p.

4. Buishand T.A. (1984). Tests for detecting a shift in the mean of hydrological time series. J Hydrol 73: 51-69.

5. Bultot F. (1957). Contribution conjointe de la température et de l'humidité de l'air au Congo Belge. Publication INEAC, communication $\mathrm{N}^{\circ} 14$.

6. INERA (Institut National pour l'Etude et la Recherche Agronomiques) (2017). Rapport annuel 2017, Centre de Yangambi. 
7. Hirsch N., Hoffman J. et Hontarrede M. (2016). Petit manuel de météo marine, Glénat (www.glenatlivres.com), 2016, 191 p. (ISBN 9782723481106).

8. Kearsley E. (2015). Carbon storage and functional diversity of tropical rainforest in the Congo basin. $\mathrm{PhD}$ thesis in applied biological sciences, Ghent University, Belgium. 183p.

9. Likoko B.A., Likoko A.G., Likoko A.B., Likoko K.J., Alongo L.S., et al., (2019a). Agricultural Monthly Rainfall Trends Disturbances in Yangambi Region, D R Congo. J Aqua Sci Oceanography 1: 105.

10. Likoko B.A., Mbifo N., Besango L., Totiwe T., Badjoko D.H et al., (2019b). Climate Change for Yangambi Forest Region, DR Congo. $J$ Aqua Sci Oceanography 1: 203.

11. Quentin M. (2018). Le réchauffement climatique. Futura, rapport IPCC 2018.

12. Thorsten P. (2018). Non-Parametric Trend Tests and Change-Point Detection. Licensed under a Creative Commons License (CC BY-ND 4.0).

13. Pettitt A.N. (1979). A non-parametric approach to the change-point problem. Appl Statist 28: 126-35.

14. Paeth H., Steger C. and Merkenschlager C. (2013). Climate change It's all about probability Vol. 67 - No. 3 - 203-222DOI: 10.3112/erdkunde.2013.03.01 ISSN 0014-0015 http://www.erdkunde.uni-bonn.de.

15. Young I.R. and Ribal, A. (2019). Multiplat form evaluation of global trends in wind speed and wave height," Science journal. science.sciencemag.org/lookup/1126/science.aav 9527 Science 364, 548-552 (2019) 10 May 2019. 